ROCKY MOUNTAIN

JOURNAL OF MATHEMATICS

Volume 25, Number 1, Winter 1995

\title{
EIGENVALUE ASYMPTOTICS FOR A NON-SELFADJOINT ELLIPTIC PROBLEM INVOLVING AN INDEFINITE WEIGHT
}

\author{
MELVIN FAIERMAN
}

\begin{abstract}
Asymptotic formulae are established for the distribution functions of the real parts of the eigenvalues of a non-selfadjoint linear elliptic boundary value problem involving an indefinite weight function under more general conditions than hitherto considered.
\end{abstract}

1. Introduction. An inspection of the literature concerning the eigenvalue asymptotics for linear elliptic boundary value problems involving an indefinite weight function shows that all of the relevant work to date has been devoted to either selfadjoint problems or nonselfadjoint problems arising from perturbations of selfadjoint ones. We refer to $[4-\mathbf{7}, \mathbf{8}, \mathbf{1 0}, \mathbf{1 1}]$, and $[\mathbf{1 3}, \mathbf{1 4}]$ for further information. We are now going to focus our attention upon the eigenvalue asymptotics for a non-selfadjoint problem which does not arise from a perturbation of a self-adjoint one.

Accordingly, we shall be concerned here with the boundary value problem

$$
\begin{gathered}
L u=\lambda \omega(x) u \text { in } \Omega, \\
B_{j} u=0 \text { on } \Gamma \quad \text { for } j=1, \ldots, m,
\end{gathered}
$$

where $L$ is a linear elliptic operator of order $2 m$ defined in a bounded region $\Omega \subset \mathbf{R}^{n}, n \geq 2$, with boundary $\Gamma$, the $B_{j}$ are linear differential operators defined on $\Gamma$, and $\omega$ is a real-valued function in $L^{\infty}(\Omega)$ which assumes both positive and negative values. Our assumptions concerning the problem (1.1-2) will be made precise in Section 2; and in particular we might mention that it will always be supposed here that $2 m>n$ and that $1 / \omega \in L^{\infty}(\Omega)$. As a consequence of our assumptions

Received by the editors on July 10, 1992 .

Copyright (C1995 Rocky Mountain Mathematics Consortium 
we are then able to appeal to the results of $[\mathbf{9}]$, wherein important information was established concerning the angular distribution of the eigenvalues of the problem (1.1-2); and in particular it was shown that the only directions of condensation of the eigenvalues of (1.1-2) were the positive and negative real axes. These results, together with the method of Carleman-Agmon, enable us in Section 3 to establish asymptotic formulae for the distribution functions of the real parts of the eigenvalues of the problem (1.1-2).

2. Preliminaries. In this section we are going to introduce our basic assumptions concerning the problem (1.1-2) and present some results from [9] which we require in the sequel. Accordingly, for $k$ a non-negative integer, let $H^{k}(\Omega)$ denote the usual Sobolev space of order $k$ related to $\mathcal{H}=L^{2}(\Omega)$ and let $(,)_{k, \Omega}$ and \|\|$_{k, \Omega}$ denote the inner product and norm, respectively, in $H^{k}(\Omega)$. We also let $($,$) and$ || $\|$ denote the inner product and norm, respectively, in $\mathcal{H}$, while if $\alpha$ denotes the multi-index $\left(\alpha_{1}, \ldots, \alpha_{n}\right)$, then we let $|\alpha|=\sum_{j=1}^{n} \alpha_{j}$ and $D^{\alpha}=D_{1}^{\alpha_{1}} \cdots D_{n}^{\alpha_{n}}$, where $D_{j}=\partial / \partial x_{j}$. Turning now to the problem (1.1-2), we henceforth suppose that

Assumption 2.1. (1) $2 m>n$;

(2) $\Omega$ is of class $C^{2 m, 1}$;

(3) $L(x, D)=\sum_{|\alpha| \leq 2 m} a_{\alpha}(x) D^{\alpha}$ is uniformly strongly elliptic in $\Omega$ with $a_{\alpha}$ real-valued if $|\alpha|=2 m$ and complex-valued otherwise and such that $a_{\alpha} \in C^{|\alpha|-1,1}(\bar{\Omega})$ for $|\alpha| \geq 1, a_{\alpha} \in L^{\infty}(\Omega)$ otherwise, wheredenotes closure;

(4) $B_{j}(x, D)=\partial^{s+j-1} / \partial \nu^{s+j-1}+\sum_{|\alpha|<s+j-1} b_{j, \alpha}(x) D^{\alpha}$ for $j=$ $1, \ldots, m$, where $s$ is an integer satisfying $0 \leq s \leq m, \partial / \partial \nu$ denotes differentiation at the boundary along a smoothly varying non-tangential direction $\nu$ (note that if we write $\partial / \partial \nu=\sum_{r=1}^{n} b_{r}(x) D_{r}$ for $x \in \Gamma$, then we require that $\left.b_{r} \in C^{2 m-2,1}(\Gamma) \cap C^{s+m-1,1}(\Gamma)\right)$, the $b_{j, \alpha}$ are complexvalued functions satisfying $b_{j, \alpha} \in C^{2 m-s-j, 1}(\Gamma) \cap C^{|\alpha|, 1}(\Gamma)$, and the summation does not appear if $s=0$ and $j=1$.

It is clear that apart from certain smoothness conditions, our assump- 
tions ensure that the boundary value problem:

$$
L u=f \quad \text { in } \Omega
$$

together with the boundary conditions (1.2) is a regular boundary value problem in the sense of $[\mathbf{1}, \mathbf{1 6}]$. Note that if $L^{\star}$ denotes the formal adjoint of $L$ and $\left\{C_{j}\right\}_{1}^{m}$ denotes a system of boundary operators adjoint to $\left\{B_{j}\right\}_{1}^{m}$ with respect to the problem $(2.1),(1.2)$ (see $[\mathbf{1 6}$, p. 121, 17]), then the formal adjoint problem of (2.1), (1.2),

$$
\begin{array}{ll}
L^{\star} u=f & \text { in } \Omega, \\
C_{j} u=0 & \text { on } \Gamma \quad \text { for } j=1, \ldots, m,
\end{array}
$$

is also a regular elliptic problem. Now let $A$ (respectively $A^{\prime}$ ) denote the operator in $\mathcal{H}$ induced by $L$ (respectively $L^{\star}$ ) and the boundary conditions (1.2) (respectively (2.2)), and where we note from [9] that $D(A)$ and $D\left(A^{\prime}\right)$ are densely defined in $\mathcal{H}$ and are closed subspaces of $H^{2 m}(\Omega)$ (here $D$ denotes domain). Then we know from $[\mathbf{9}]$ that $A^{\prime}=A^{\star}$, where $A^{\star}$ denotes the Hilbert space adjoint of $A$, and that $A$ and $A^{\star}$ are Fredholm operators [15, p. 230].

Turning next to our assumptions concerning $\omega(x)$, let

$$
\begin{aligned}
& \Omega^{+}=\{x \in \Omega ; \omega(x)>0\}, \\
& \Omega^{-}=\{x \in \Omega ; \omega(x)<0\}, \\
& \Omega^{0}=\{x \in \Omega ; \omega(x)=0\} .
\end{aligned}
$$

Assumption 2.2. We henceforth suppose that:

(1) $\left|\Omega^{ \pm}\right|>0$ and $\left|\Omega^{0}\right|=0$, where || denotes $n$-dimensional Lebesgue measure;

(2) $\mid \Omega^{ \pm} \backslash$ int $\Omega^{ \pm} \mid=0$, where int stands for interior;

(3) int $\Omega^{+}$(respectively int $\Omega^{-}$) is the union of a finite number of nonempty disjoint regions, say $\left\{\Omega_{r}^{+}\right\}$(respectively $\left\{\Omega_{r}^{-}\right\}$), each of which is of class $C^{2 m-1,1}$ and in each of which $\omega(x)$ is uniformly continuous and $|\omega(x)|$ has a positive infimum;

(4) each component $\Gamma_{r j}^{+}$of $\partial \Omega_{r}^{+}$( $\partial$ is boundary) is either a component of $\Gamma$ or is contained in $\Omega$, and in this latter case $\Gamma_{r j}^{+}$is also either a component of $\partial \Omega_{s}^{+}$for some $s \neq r$, or a component of $\partial \Omega_{s}^{-}$for some $s$; 
(5) the same hypothesis as in (4) holds with the superscripts + and - interchanged.

Let $T$ denote the operator of multiplication in $\mathcal{H}$ induced by $\omega$. Then the eigenvalue problem (1.1-2) can be formulated from a purely operator-theoretic point of view, namely as the eigenvalue problem in $\mathcal{H}: T^{-1} A u=\lambda u$. Hence we call $\lambda$ an eigenvalue of the problem (1.1-2) if $\lambda$ is an eigenvalue of $T^{-1} A$; and by the multiplicity (geometric or algebraic) of the eigenvalue $\lambda$ we mean it multiplicity as an eigenvalue of $T^{-1} A$. We shall also refer to the principal vectors and principal subspace for the eigenvalue $\lambda$ of $T^{-1} A$ as those for the eigenvalue $\lambda$ of (1.1-2); and if $\lambda$ is an eigenvalue of (1.1-2), then we henceforth let $M_{\lambda}$ denote the principal subspace corresponding to $\lambda$.

Assumption 2.3. We suppose from now on that $\rho\left(T^{-1} A\right) \neq \varnothing$.

We refer to [9] for a detailed discussion of Assumption 2.3. Moreover, from $[\mathbf{9}]$ we also know that (here, when we refer to the spectrum of the problem $(1.1-2)$, we mean the spectrum of $T^{-1} A$ ),

Theorem 2.1. The spectrum of the problem (1.1-2) consists solely of eigenvalues of finite algebraic multiplicity which form a denumerably infinite subset of $\mathbf{C}$ having no finite points of accumulation. Furthermore, for any $\varepsilon$ satisfying $0<\varepsilon<\pi / 2$, there are infinitely many eigenvalues of (1.1-2) lying in each of the sectors: $|\arg \lambda|<\varepsilon$ and $|\arg \lambda-\pi|<\varepsilon$, while there are at most a finite number of eigenvalues in each of the sectors: $\varepsilon \leq \arg \lambda \leq \pi-\varepsilon$ and $-\pi+\varepsilon \leq \arg \lambda \leq-\varepsilon$.

In light of Theorem 2.1, we see that there is no loss of generality in introducing

Assumption 2.4. We suppose from now on that $0 \in \rho\left(T^{-1} A\right)$.

Definition 2.1. Let $X$ be a complex Hilbert space and $S$ a linear operator in $X$. Then the set of all non-zero complex numbers $\lambda$ such that $I-\lambda S$ has an inverse in $\mathcal{L}(X)$ is called the modified resolvent set 
of $S$ and is denoted by $\rho_{m}(S)$. For $\lambda \in \rho_{m}(S)$ we let $S_{\lambda}=S(I-\lambda S)^{-1}$ and call $S_{\lambda}$ the modified resolvent of $S$. A complex number $\lambda$ is called a characteristic value of $S$ if there exists a $u \neq 0$ in $D(S)$ such that $(I-\lambda S) u=0 ; u$ is called a characteristic vector of $S$ corresponding to $\lambda$. If $\lambda$ is a characteristic value of $S$, then a non-zero vector $u$ is called a generalized characteristic vector of $S$ corresponding to $\lambda$ if for some $p \in \mathbf{N}, u \in D\left(S^{p}\right)$ and $(I-\lambda S)^{p} u=0$. The set consisting of all generalized characteristic vectors of $S$ corresponding to $\lambda$ together with the zero vector in $X$ is a subspace of $X$ which we denote by $\mathcal{G}_{\lambda}(S, X)$. Finally, the ray $\arg \lambda=\theta$ in the complex $\lambda$-plane is said to be a ray of minimal growth of $S_{\lambda}$ if for all $\lambda$ on the ray, with $|\lambda|$ sufficiently large, we have $\lambda \in \rho_{m}(S)$ and $\left\|S_{\lambda}\right\|_{X} \leq c|\lambda|^{-1}$, where $c$ denotes a positive constant and \|\|$_{X}$ denotes the norm in $\mathcal{L}(X)$.

It follows from what has been said above that $0 \in \rho(A)$ and that $A^{-1}$ is compact. Hence let us now introduce in $\mathcal{H}$ the compact operator $K=A^{-1} T$. Then from $[\mathbf{9}]$ we have

Theorem 2.2. $\lambda$ is an eigenvalue of the problem (1.1-2) if and only if $\lambda$ is a characteristic value of $K$. Moreover, if $\lambda$ is an eigenvalue of (1.1-2), then $M_{\lambda}=\mathcal{G}_{\lambda}(K, \mathcal{H})$. Finally, every ray $\arg \lambda=\theta$ in the complex $\lambda$-plane for which $\theta \neq k \pi, k \in \mathbf{Z}$, is a ray of minimal growth of $K_{\lambda}$.

3. Main results. We are now going to use the foregoing information to derive our main results. Accordingly, since $A^{-1}$ and $A^{\star-1}$ both map $\mathcal{H}$ into $H^{2 m}(\Omega)$, an easy argument involving the polar decomposition of $A^{-1}$ and the results of [1] shows that $K$ is of trace class (or nuclear in the terminology of [12]). Furthermore, we know from $[\mathbf{3}]$ that $K$ is an integral operator with kernel $G(x, y) \omega(y)$, where $G(x, y)$ is continuous and bounded in $\Omega \times \Omega$. Now for $\theta \in \mathbf{R}$ such that $\theta \neq k \pi$ for $k \in \mathbf{Z}$, let $\Xi(\theta)$ denote the ray in the complex $\lambda$-plane emanating from the origin and making an angle $\theta$ with the positive real axis and for $a \geq 0$, let $\Xi(\theta, a)=\{\lambda \in \mathbf{C}|\lambda \in \Xi(\theta),| \lambda \mid \geq a\}$. We henceforth suppose that $a$ chosen large enough so that $\Xi(\theta, a) \subset \rho_{m}(K)$. Then for $\lambda \in \Xi(\theta, a)$, it is clear that $K_{\lambda}$ is of trace class, while from [3] we know that $K_{\lambda}$ is an integral operator with kernel $G_{\lambda}(x, y) \omega(y)$, where $G_{\lambda}$ is continuous 
and bounded in $\Omega \times \Omega$, and that in this region we have

$$
\left|G_{\lambda}(x, y)\right| \leq c|\lambda|^{-1+n / 2 m},
$$

where $c$ denotes a positive constant not depending upon $\lambda, x$, nor $y$. Also if $L_{0}(x, D)$ denotes the principal part of $L(x, D)$, then

Theorem 3.1. If $x^{0} \in \Omega_{r}^{ \pm}$, then

$$
G_{\lambda}\left(x^{0}, x^{0}\right) \omega\left(x^{0}\right)=|\lambda|^{-1+n / 2 m}\left[m_{\theta}\left(x^{0}\right)+o(1)\right]
$$

as $|\lambda| \rightarrow \infty, \lambda \in \Xi(\theta, a)$, where

$$
m_{\theta}\left(x^{0}\right)=(2 \pi)^{-n} \int_{\mathbf{R}^{n}}\left[\omega\left(x^{0}\right)^{-1} L_{0}\left(x^{0}, i \xi\right)-e^{i \theta}\right]^{-1} d \xi .
$$

Proof. The theorem can readily be established by arguing as in the proof of Theorem 7.1 of $[\mathbf{3}]$ and making use of the facts that $(A-\lambda T) K_{\lambda} T^{-1} f=f$ and $\left(A^{\star}-\bar{\lambda} T\right)\left(K_{\lambda} T^{-1}\right)^{\star} f=f$ for $f \in \mathcal{H}$, where ${ }^{\star}$ denotes adjoint.

Finally let $\left\{\lambda_{j}\right\}_{1}^{\infty}$ denote the eigenvalues of the problem (1.1-2) counted according to algebraic multiplicity, and for $\lambda \geq 0$ let $N_{+}(\lambda)$ denote the number of eigenvalues $\lambda_{j}$ for which $0 \leq \operatorname{Re} \lambda_{j} \leq \lambda$, while for $\lambda>0$ let $N_{-}(\lambda)$ denote the number of eigenvalues $\lambda_{j}$ for which $-\lambda \leq$ $\operatorname{Re} \lambda_{j}<0$ and put $N_{-}(0)=0$. Also let $N(\lambda)=N_{+}(\lambda)+N_{-}(\lambda)$ for $\lambda \geq 0$ so that $N(\lambda)$ is the number of eigenvalues $\lambda_{j}$ for which $\left|\operatorname{Re} \lambda_{j}\right| \leq$ $\lambda$. Then putting $\omega^{+}(x)=\max \{\omega(x), 0\}, \omega^{-}(x)=\max \{-\omega(x), 0\}$, and $\tau(x)=\left|\left\{\xi \in \mathbf{R}^{n} \mid 0<L_{0}(x, i \xi)<1\right\}\right|$, where here || denotes $n$-dimensional Lebesgue measure, our main result is

Theorem 3.2. It is the case that

$$
\begin{gathered}
N_{ \pm}(\lambda)=c_{ \pm} \lambda^{n / 2 m}+o\left(\lambda^{n / 2 m}\right) \quad \text { as } \lambda \rightarrow \infty, \\
N(\lambda)=c \lambda^{n / 2 m}+o\left(\lambda^{n / 2 m}\right) \quad \text { as } \lambda \rightarrow \infty,
\end{gathered}
$$


where

$$
\begin{aligned}
c_{ \pm} & =(2 \pi)^{-n} \int_{\Omega}\left(\omega^{ \pm}(x)\right)^{n / 2 m} \tau(x) d x, \\
c & =c_{+}+c_{-}=(2 \pi)^{-n} \int_{\Omega}|\omega(x)|^{n / 2 m} \tau(x) d x .
\end{aligned}
$$

Proof. It follows from (3.1), Theorem 3.1, and the Lebesgue dominated convergence theorem that

$$
\int_{\Omega} G_{\lambda}(x, x) \omega(x) d x=|\lambda|^{-1+n / 2 m}\left[\int_{\Omega} m_{\theta}(x) d x+o(1)\right]
$$

as $|\lambda| \rightarrow \infty, \lambda \in \Xi(\theta, a)$. On the other hand, it follows from Theorem 2.2 and arguments similar to those used in the proof of Theorem 13.10 of $[\mathbf{2}$, p. 227] that

$$
\int_{\Omega} G_{\lambda}(x, x) \omega(x) d x=\sum_{j=1}^{\infty}\left(\lambda_{j}-\lambda\right)^{-1},
$$

and hence we conclude that

$$
\sum_{j=1}^{\infty}\left(\lambda_{j}-\lambda\right)^{-1}=|\lambda|^{-1+n / 2 m}\left[\int_{\Omega} m_{\theta}(x) d x+o(1)\right]
$$

as $|\lambda| \rightarrow \infty, \lambda \in \Xi(\theta, a)$. Taking $\theta=\pi / 2, \lambda=i t$, we see from (3.2) that

$$
\begin{aligned}
\sum_{\alpha=1}^{\infty}\left(\lambda_{j}-i t\right)^{-1}= & \left(\int_{\Omega} m_{\pi / 2}(x) d x\right) t^{-1+n / 2 m} \\
& +o\left(t^{-1+n / 2 m}\right) \text { as } t \rightarrow \infty .
\end{aligned}
$$

Hence if we let $\lambda_{j}=\mu_{j}+i \nu_{j}$ for $j \geq 1$ and bear in mind Theorem 2.1 and the fact that $K$ is of trace class, then it is easy to deduce from (3.3) that

$$
\begin{aligned}
\sum_{j=1}^{\infty}\left(\mu_{j}-i t\right)^{-1}= & \left(\int_{\Omega} m_{\pi / 2}(x) d x\right) t^{-1+n / 2 m} \\
& +o\left(t^{-1+n / 2 m}\right) \quad \text { as } t \rightarrow \infty
\end{aligned}
$$


We can now appeal to a Tauberian theorem of Hardy and Littlewood and argue with (3.4) precisely as in the proof of Theorem 14.6 of $[\mathbf{2}$, p. 250] to arrive at the conclusions of the theorem.

\section{REFERENCES}

1. S. Agmon, On the eigenfunctions and on the eigenvalues of general elliptic boundary value problems, Comm. Pure Appl. Math. 15 (1962), 119-147.

2. - Lectures on elliptic boundary value problems, Van Nostrand, Princeton, NJ, 1965.

3. - On kernels, eigenvalues, and eigenfunctions of operators related to elliptic problems, Comm. Pure Appl. Math. 18 (1965), 627-663.

4. M.S. Birman and M.Z. Solomjak, Spectral asymptotics of nonsmooth elliptic operators II, Trans. Moscow Math. Soc. 28 (1973), 1-32.

5. - Asymptotic behaviour of the spectrum of differential equations, J. Soviet Math. 12 (1974), 247-282.

6. - Asymptotics of the spectrum of variational problems on solutions of elliptic equations, Siberian Math. J. 20 (1979), 1-15.

7. - Quantitative analysis in Sobolev imbedding theorems and applications to spectral theory, Amer. Math. Soc. Transl. 114 (1980).

8. M. Faierman, On the eigenvalues of nonselfadjoint problems involving indefinite weights, Math. Ann. 282 (1988), 369-377.

9. — Non-selfadjoint elliptic problems involving an indefinite weight, Comm. Partial Differential Equations 15 (1990), 939-982.

10. J. Fleckinger and M.L. Lapidus, Eigenvalues of elliptic boundary value problems with an indefinite weight function, Trans. Amer. Math. Soc. 295 (1986), 305-324.

11. - Remainder estimates for the asymptotics of elliptic eigenvalue problems with indefinite weights, Arch. Rational Mech. Anal. 98 (1987), 329-356.

12. I.C. Gohberg and M.G. Krein, Introduction to the theory of linear nonselfadjoint operators, Amer. Math. Soc., Providence, 1969.

13. P. Hess, On the asymptotic distribution of eigenvalues of some nonselfadjoint problems, Bull. London Math. Soc. 18 (1986), 181-184.

14. - On the spectrum of elliptic operators with respect to indefinite weights, Linear Algebra Appl. 84 (1986), 99-109.

15. T. Kato, Perturbation theory for linear operators, 2nd ed., Springer-Verlag, New York, 1976.

16. J.L. Lions and E. Magenes, Non-homogeneous boundary value problems and applications, Vol. I, Springer-Verlag, New York, 1972. 
17. M. Schechter, General boundary value problems for elliptic partial differential equations, Comm. Pure Appl. Math. 12 (1959), 457-482.

Department of Mathematics, University of the Witwatersrand, JohanNesburg, Wits 2050, South Africa. 